\title{
Mesoscopic domain average engineering simulations of ferroelectric perovskites compared with multidomain group theoretical predictions
}

\author{
Brandon J. Keith \\ Dorian M. Hatch \\ hatchd@byu.edu
}

Follow this and additional works at: https://scholarsarchive.byu.edu/facpub

Part of the Astrophysics and Astronomy Commons, and the Physics Commons

\section{Original Publication Citation}

Keith, Brandon J. and Dorian M. Hatch."Mesoscopic domain average engineering simulations of ferroelectric perovskites compared with multidomain group theoretical predictions." Journal of Applied Physics 1 (26).

\section{BYU ScholarsArchive Citation}

Keith, Brandon J. and Hatch, Dorian M., "Mesoscopic domain average engineering simulations of ferroelectric perovskites compared with multidomain group theoretical predictions" (2006). Faculty Publications. 299.

https://scholarsarchive.byu.edu/facpub/299 


\title{
Mesoscopic domain average engineering simulations of ferroelectric perovskites compared with multidomain group theoretical predictions
}

\author{
J. Brandon Keith ${ }^{\text {a) }}$ and Dorian M. Hatch \\ Department of Physics and Astronomy, Brigham Young University, Provo, Utah 84604
}

(Received 7 February 2006; accepted 19 June 2006; published online 14 August 2006)

\begin{abstract}
Engineered, multidomain ferroelectrics are of current interest for mechatronic and optical applications. Previously [D. M. Hatch et al., J. Appl. Phys. 94, 5220 (2003)], we presented a set of tables/symmetry rules showing all possible domain average engineered multidomain structures arising from [100]-, [110]-, and [111]-ordering ferroelectric transitions from a $P m \overline{3} m$ perovskite structure. In this work we test those predictions against three-dimensional mesoscopic fast quench simulations under various electric/stress field combinations and find excellent agreement with multidomain group theory. These simulations use a Langevin strain-displacement/polarization evolution strategy and are important to developing processing conditions for materials with precise domain structures. () 2006 American Institute of Physics. [DOI: 10.1063/1.2234557]
\end{abstract}

\section{INTRODUCTION}

Ferroelectric ceramics are used in a variety of optoelectronic and mechatronic devices due to their pronounced dielectric, optical, piezoelectric, and pyroelectric properties. Mechatronic devices include bending actuators, flow and level control products, nebulizers (fluid atomizers), ultrasonic transducers, buzzers and ignitors, ultrasonic welding components, superpiezospeakers, microhydraulic transducers, large force/displacement microelectromechanical system (MEMS) microactuators, ${ }^{1}$ and flapping wing actuators of micromechanical flying insects. ${ }^{2}$ Optoelectronic applications include periodically poled crystals for frequency conversion, ${ }^{3}$ electro-optic Bragg switches, ${ }^{4}$ and complex structures used for photonic band gap devices. ${ }^{5}$ Material properties are related to domain structures. If one can design or control domain formation, one should be able to take full advantage of ferroelectric materials in fabricating nextgeneration optical and mechatronic devices.

There has been much previous work on modeling multidomain ferroelectric structures. Cao and co-workers have proposed a three-dimensional Landau-Ginzburg model to describe the tetragonal twin microstructures in ferroelectrics, and have studied boundary condition effects on ferroelectric transitions, the influence of dipolar defects on ferroelectric switching, the influence of system size, the effect of surface induced nucleation of ferroelastic domains on polarization switching, and the piezoelectric response of engineered domains. ${ }^{6-10}$ Nambu and Sagala simulated two-dimensional (2D) domain structures in ferroelectrics with consideration of multiple dipole-dipole-elastic contributions. ${ }^{11}$ Chen and coworker have compared the influence of long-range elastic, dipole, and external field contributions to domain morphology and have examined polarization switching. ${ }^{12-14}$ Bhattacharya and co-worker have developed a potential gradient flow model of ferroelectrics to simulate domain switching, grain boundaries, and defect pinning. ${ }^{15,16}$

\footnotetext{
${ }^{a)}$ Author to whom correspondence should be addressed; electronic mail: jbrkeith@gmail.com
}

In this work we simulate domain average engineering (DAE) of perovskite ferroelectrics. Domain engineering has been discussed at length ${ }^{17-20}$ and refers to at least two kinds of structural descriptions. The first, domain geometry engineering, is a description including the details of geometrical configurations in space, twinning patterns, and domain wall orientation and positioning. The second, domain average engineering, deals with an average of domain configurations without considering spatial occupation details. Allowable symmetry operations on such structures refer to domain volume fractions and may not bring the local structure back to itself. Group theoretical considerations leading to domain average engineered structures were discussed in a previous study. ${ }^{21}$

Reference 21 also derived all possible multidomain structures arising after a ferroelectric transition from the paraelectric $P m \overline{3} m$ perovskite structure to the [100]-ordering $P 4 \mathrm{~mm}$ structure, the [111]-ordering $R 3 \mathrm{~m}$ structure, and the [110]-ordering Amm2 structure. This was done by finding combinations of external fields which induce each multidomain symmetry. Here we simulate a diverse cross section of multidomain symmetries for each of these ordering structures using a mesoscopic phase field model. This model is similar to those in previous studies but also has some important advantages which are discussed Secs. II A and II B. These simulations are done without any inclusion of the symmetries in Ref. 21. Each multidomain structure is a product only of the external stress/electric fields and the underlying thermodynamics / kinetics.

The outline of the balance of the paper is as follows. Section II A describes our coarse-grained model and an improved simulation strategy. Section II B details some of the numerical issues encountered in simulating DAE and their resolution. Section III A illustrates our simulation methodology on three types of proper ferroelectric perovskite multidomain structures paralleling previous group theoretical derivations. Implications and future work are discussed in Secs. III B and III C. 


\section{MODEL DEVELOPMENT}

\section{A. Phase field theory}

As an apropos mesoscopic model we have chosen phase field theory, ${ }^{12}$ which is based on fundamental principles of thermodynamics and kinetics and which provides a powerful method for predicting the temporal evolution of microstructure. This is done by solving kinetic equations of spatially inhomogeneous order parameters without any prior assump- tions of transient microstructure. The phase field, free energy functional is

$$
F[P(\mathbf{r}), u(\mathbf{r})]=\int_{\Omega} d \mathbf{r}\left(f_{P}+f_{u}+f_{c}+f_{E}+f_{\mathrm{dep}}\right) .
$$

$f_{P}$ is the Landau-Ginzburg free energy functional for polarization (including gradient terms),

$$
\begin{aligned}
f_{P}= & \alpha_{1}\left(P_{1}^{2}+P_{2}^{2}+P_{3}^{2}\right)+\alpha_{11}\left(P_{1}^{4}+P_{2}^{4}+P_{3}^{4}\right)+\alpha_{12}\left(P_{1}^{2} P_{2}^{2}+P_{2}^{2} P_{3}^{2}+P_{1}^{2} P_{2}^{2}\right)+\alpha_{111}\left(P_{1}^{6}+P_{2}^{6}+P_{3}^{6}\right)+\alpha_{112}\left[P_{1}^{4}\left(P_{2}^{2}+P_{3}^{2}\right)\right. \\
& \left.+P_{2}^{4}\left(P_{1}^{2}+P_{3}^{2}\right)+P_{3}^{4}\left(P_{1}^{2}+P_{2}^{2}\right)\right]+\alpha_{123} P_{1}^{2} P_{2}^{2} P_{3}^{2}+\cdots+\frac{1}{2} G_{11}\left(P_{1,1}^{2}+P_{2,2}^{2}+P_{3,3}^{2}\right)+G_{12}\left(P_{1,1}^{2} P_{2,2}^{2}+P_{2,2}^{2} P_{3,3}^{2}+P_{1,1}^{2} P_{3,3}^{2}\right) \\
& +G_{44}\left[\left(P_{1,2}+P_{2,1}\right)^{2}+\left(P_{2,3}+P_{3,2}\right)^{2}+\left(P_{1,3}+P_{3,1}\right)^{2}\right]+\frac{1}{2} G_{44}^{\prime}\left[\left(P_{1,2}-P_{2,1}\right)^{2}+\left(P_{2,3}-P_{3,2}\right)^{2}+\left(P_{1,3}-P_{3,1}\right)^{2}\right]+\cdots .
\end{aligned}
$$

The $\alpha$ coefficients are related to dielectric stiffness. ${ }^{22}$ Trailing dots represent possible higher order terms. The $G$ coefficients can be related to domain size, domain wall thickness, and domain orientation. $f_{u}$ is the elastic energy. As a first approximation we include only second order terms and use the infinitesimal Lagrangian strain tensor $\varepsilon_{i j}=\frac{1}{2}\left(u_{i, j}+u_{j, i}\right)$ where $u_{i}$ is the displacement,

$$
\begin{aligned}
f_{u}= & \frac{1}{2} c_{11}\left(\varepsilon_{11}^{2}+\varepsilon_{22}^{2}+\varepsilon_{33}^{2}\right)+c_{12}\left(\varepsilon_{11} \varepsilon_{22}+\varepsilon_{22} \varepsilon_{33}+\varepsilon_{11} \varepsilon_{33}\right) \\
& +2 c_{44}\left(\varepsilon_{12}^{2}+\varepsilon_{23}^{2}+\varepsilon_{13}^{2}\right) .
\end{aligned}
$$

Higher order terms, gradient terms, and the finite Lagrangian strain tensor would increase accuracy away from the phase transition and at larger strains. Expansions in polarization or strain and their derivatives are automatically generated to an arbitrary order for any of the 230 space groups using ISOTROPY. ${ }^{23} f_{c}$ is a coupling term between polarization and strain,

$$
\begin{aligned}
f_{c}= & -k_{11}\left(\varepsilon_{11} P_{1}^{2}+\varepsilon_{22} P_{2}^{2}+\varepsilon_{33} P_{3}^{2}\right)-k_{12}\left[\varepsilon_{11}\left(P_{2}^{2}+P_{3}^{2}\right)\right. \\
& \left.+\varepsilon_{22}\left(P_{1}^{2}+P_{3}^{2}\right)+\varepsilon_{33}\left(P_{1}^{2}+P_{2}^{2}\right)\right] \\
& -2 k_{44}\left(\varepsilon_{12} P_{1} P_{2}+\varepsilon_{13} P_{1} P_{3}+\varepsilon_{23} P_{2} P_{3}\right)+\cdots
\end{aligned}
$$

where $k_{i}$ are the electromechanical coupling coefficients. $f_{E}$ is an electric field interaction term,

$$
f_{E}=-\mathbf{E} \cdot \mathbf{P},
$$

and $f_{\text {dep }}$ is a deopolarization term,

$$
f_{\text {dep }}=-\mathbf{E}_{\text {dep }} \cdot \mathbf{P},
$$

which is discussed further in Sec. II B.

Phase field theory evolves the system's free energy in time using a long-wavelength largest eigenmode formalism. ${ }^{24}$ This is synonymous to Langevin dynamics, time-dependent Ginzburg-Landau theory, and the Allen-Cahn equation for nonconserved order parameter evolution. It is a well-established formalism for equilibrium relaxation ${ }^{12}$ and has been used frequently on ferroelectrics. ${ }^{7-11,13,14,25,26}$ In contrast to other ferroelectric theoretical treatments, how- ever, we evolve both primary $\left(P_{i}\right.$ polarization) and secondary $\left(u_{i}\right.$ strain displacement) order parameters in a coupled set of equations,

$$
\begin{aligned}
& \frac{\partial}{\partial t} P_{i}(\mathbf{r}, t)=-\Gamma^{P} \frac{\delta F}{\delta P_{i}(\mathbf{r}, t)}+\xi_{i}^{P}(\mathbf{r}, t), \\
& \frac{\partial}{\partial t} u_{i}(\mathbf{r}, t)=-\Gamma^{u} \frac{\delta F}{\delta u_{i}(r, t)}+\xi_{i}^{u}(\mathbf{r}, t) .
\end{aligned}
$$

$\Gamma^{o p}$ is a kinetic coefficient and $o p$ is either $u$ or $P . \xi_{i}^{o p}(\mathbf{r}, t)$ is a Gaussian random force where

$$
\begin{aligned}
& \left\langle\xi_{i}^{o p}(\mathbf{r}, t)\right\rangle=0, \\
& \left\langle\xi_{i}^{o p}(\mathbf{r}, t) \xi_{j}^{o p}\left(\mathbf{r}^{\prime}, t^{\prime}\right)\right\rangle=2 k_{B} T \Gamma^{o p} \delta_{i j} \delta\left(\mathbf{r}-\mathbf{r}^{\prime}\right) \delta\left(t-t^{\prime}\right) .
\end{aligned}
$$

These Langevin equations are frequently used to evolve strain, ${ }^{27-30}$ and occasionally Newtonian dynamical equations are used to evolve strain displacements. ${ }^{31-35}$ However, it is equally valid to use the Langevin formalism to evolve strain displacements directly. This latter approach implicitly satisfies compatibility constraints ${ }^{34}$ and simplifies a real space simulation.

As this approach is different from those reviewed in the Introduction we comment once again on some of its attributes. Most ferroelectric simulations employ instantaneous long-range elastic contributions to the free energy, meaning lattice strains are treated as a secondary order parameter and are obtained via a variational procedure. The displacements are substituted into the kinetic equations for polarization evolution resulting in the so-called "adiabatic approximation" which produces long-range elastic interactions in the free energy and instantaneous mechanical equilibrium. However, it should be clearly understood that in Eqs. (7) mechanical equilibrium is not enforced at each time step. Strictly speaking, enforced mechanical equilibrium is nonphysical since the real system requires a finite amount of time to relax to mechanical equilibrium. However, as strain modes operate at much faster frequencies than polarization 
TABLE I. Polarization expansion coefficients.

\begin{tabular}{lrrrrrr}
\hline \hline & $\alpha_{1}$ & $\alpha_{11}$ & $\alpha_{12}$ & $\alpha_{111}$ & $\alpha_{112}$ & $\alpha_{123}$ \\
\hline$(a, 0,0)$ & -1.00 & -0.50 & 9.00 & 0.80 & 4.00 & 1.00 \\
$(a, a, a)$ & -6.45 & -2.98 & -1.55 & 6.46 & 1.06 & -2.04 \\
$(a, a, 0)$ & -6.88 & -2.69 & 5.09 & 2.69 & 0.35 & 3.66 \\
\hline \hline
\end{tabular}

modes, it is common to make this approximation. Equations (7) remove this approximation by evolving strain displacement with a separate kinetic coefficient. However, the real advantage is they remove the long-range integrals; coupling to mechanical modes occurs naturally through the differential equations or free energy functional during microstructure evolution.

Another attribute of this model is that it is based on the well-known Landau theory of phase transitions ${ }^{36,37}$ which has proven to be a simple yet effective model of mesoscopic phenomena based on symmetry-adapted polynomial expansions in primary and secondary order parameters and their gradients. It has been used frequently in ferroelectric simulations. Some ferroelectric treatments discussed in Sec. I include additional long-range dipole terms or solve Maxwell's equations. Although these studies may capture certain physical phenomena a Landau-based theory cannot, these approaches are not particularly necessary in a study which compares traditional mesoscopic simulations of prototypical ferroelectrics with group theoretical predictions of domain morphology. Additionally, we have found little difference when including these terms for the particular simulations presented here; the gradient terms sufficiently take into account interactions between different regions of the system. We now turn to the methods used to solve this set of differential equations.

\section{B. Numerical implementation}

There are several ways to obtain the coefficients for the free energy functional. The most common method is to obtain them from experimental measurements. However, this creates a dependence on empirical accuracy, cripples design efforts, and nullifies predictive power outside experimental ranges. Another way is by first principles calculation which has had encouraging results ${ }^{38-44}$ but is still under development due to approximations when transferring information from a $0 \mathrm{~K}$ energy calculation to a finite temperature free energy functional. Phase transition temperatures, for example, can be off by hundreds of Kelvin. ${ }^{45}$ Another option for determining potential coefficients is to map out distinct solutions or crystallographic structures for all values of the potential parameters. These maps are called global phase diagrams (GPDs) and have been discussed in detail for molecular crystals. ${ }^{46-48}$ GPDs are intended to work in conjunction with first principles calculations as a tool for crystal engineering. As we seek to illustrate mesoscopic phenomena for prototypical classes of materials, it is sufficient to find a representative potential for each prototype using the techniques of GPDs. ${ }^{48}$ These coefficients are shown in Table I for the types of ferroelectric transitions discussed in Ref. 21, although coefficients for the $(a, 0,0)$ solution have been taken
TABLE II. Elastic coefficients and polarization-elastic coupling coefficients.

\begin{tabular}{rrrrrr}
\hline \hline$c_{11}$ & $c_{12}$ & $c_{44}$ & $k_{11}$ & $k_{12}$ & $k_{44}$ \\
\hline 2.75 & 1.79 & 0.543 & 0.142 & -0.0074 & 0.0157 \\
\hline \hline
\end{tabular}

from the literature. ${ }^{13}$ Elastic/coupling coefficients are less important in this study and so a single set, shown in Table II, was used in each case.

GPD techniques do not apply to determination of the gradient coefficients, however, which do not alter the crystalline phase but only the microscopic texture. Thus we discuss our technique for their determination. First, taking the functional derivatives in Eq. (7) reduces the number of gradient coefficients to three, $g_{11}=G_{11}, g_{12}=G_{12}+G_{44}-G_{44}^{\prime}$, and $g_{44}=G_{44}+G_{44}^{\prime}$, as shown in Appendix A. Positive gradient coefficients describe penalties associated with changes in the microscopic texture of the material. Thus with increasing magnitude they cause larger domains to form, whose shape depends on the polarization gradients with which they are coupled. Negative gradient coefficients describe penalties for uniformity in texture and so are unphysical. Estimates of the magnitude of these coefficients can be found in the literature, but for our purposes we modify these values slightly to accommodate finite size effects in our simulation box.

Ideally DAE takes place in an infinite single crystal since the initial state postulated by DAE should have equal domain volume fractions and zero total polarization. This is impossible since any numerical treatment must use finite computational cells which typically produce somewhat unequal domain volume fractions. We adopt two measures to overcome this deficiency. (1) Scale the gradient coefficients ( $g$ 's) so domain sizes are smaller than typical ferroelectrics. This is computationally justifiable since it is tantamount to an increased coarse graining of the mesoscopic model and using fewer mesh points in each domain. From this perspective domain sizes do not shrink but rather the size of the single crystal increases. It is physically justified because in a fast quench kinetic regime thermal motion effectively weakens these coefficients such that smaller domain structures are produced before being frozen in place. (2) A depolarization term is added to the free energy functional as mentioned in Sec. II A to maintain the net polarization of the crystal approximately near zero. Our treatment of depolarization does this in such a way as to equalize domain volume fractions as much as possible. It begins by postulating an overall depolarizing electric field proportional to the weighted sum of all other domain solution directions,

$$
\mathbf{E}_{\mathrm{dep}} \propto \sum_{d}\left(N_{d}-\bar{N}\right) \mathbf{d},
$$

where $d$ indexes the possible domains for a given solution type, $\mathbf{d}$ is a unit vector in that specific domain orientation (i.e., [100],[010],... for the tetragonal prototype), and

$$
N_{d}=\sum_{i} i \quad \text { where } P_{i} \in d,
$$

which is the number of polarizations most closely oriented along the polarization direction of a given domain $d$. The $i$ 
TABLE III. 5 of the 12 possible distinct multidomain sets for ferroelectric ordering along [100]. Only nonzero contributions of the order parameters are shown in columns $3,4,5$.

\begin{tabular}{llllll}
\hline \hline Domain set & \multicolumn{1}{c}{ Group } & \multicolumn{1}{c}{$\Gamma_{4}^{-}$} & $\Gamma_{3}^{+}$ & $\Gamma_{5}^{+}$ & \multicolumn{1}{c}{ Connected set } \\
\hline$(a, a, a, a, a, a)$ & $m \overline{3} m$ & & & $(1,2,3,4,5,6)$ \\
$(a, a, b, b, a, a)$ & $4_{z} / m_{x} m_{x y} m_{z}$ & & $(a, 0)$ & & $(1,2,5,6)$ \\
$(a, b, a, b, a, b)$ & $3_{x y z} m_{\overline{x y}}$ & $(a, a, a)$ & & $(a, a, a)$ & $(1,3,5)$ \\
$(a, a, b, a, a, a)$ & $4_{z} m_{x} m_{x y}$ & $(0,0, a)$ & $(a, 0)$ & & $(3)$ \\
$(a, a, b, c, c, b)$ & $m_{y z} m_{x} 2_{\overline{y z}}$ & $(0, a,-a)$ & $(a, \sqrt{3} a)$ & $(0, a, 0)$ & $(3,6)$ \\
\hline \hline
\end{tabular}

index goes over all space which is discretized, each vertex containing one polarization $P_{i} . \bar{N}$ is the average number of polarizations belonging to a given domain $d$ were all domains exactly equal ( $N_{\text {tot }} /$ number of domains). The magnitude of $\mathbf{E}_{\text {dep }}$ is variable and for numerical stability reasons is set so $f_{\text {dep }}$ is approximately equal to other contributions, $f_{P}$, $f_{u}$, and $f_{c}$, in the free energy.

Effectively $\mathbf{E}_{\text {dep }}$ pushes each domain $d$ so $N_{d}$ approaches $\bar{N}$. Even with this push domain sizes will not generally be equal since contributions to $\mathbf{E}_{\text {dep }}$ from opposite domains (i.e., $[100]$ and $[\overline{100}])$ almost exactly cancel each other leading to nearly equal opposite domain volume fractions but only somewhat equal nonopposite domain fractions. This is discussed further in Sec. III A 1.

To use group theoretical guidance we must symmetry adapt our potential to space group irreducible representations (IRs). We apply a symmetry transformation matrix $\stackrel{\leftrightarrow}{T}$ to the strain tensor, $\mathbf{e}=\stackrel{\leftrightarrow}{T} \cdot \boldsymbol{\varepsilon}$, such that, using the notation of Ref. 49 , $e_{1}$ transforms like a $\Gamma_{1}^{+}$distortion (volumetric strain), $\left(e_{2}, e_{3}\right)$ like a $\Gamma_{3}^{+}$distortion (deviatoric strain), and $\left(e_{4}, e_{5}, e_{6}\right)$ like a $\Gamma_{5}^{+}$distortion (shear strain), where

$$
\begin{aligned}
& e_{1}=\varepsilon_{11}+\varepsilon_{22}+\varepsilon_{33}, \\
& e_{2}=\frac{1}{2}\left(\varepsilon_{11}-\varepsilon_{22}\right), \\
& e_{3}=\frac{1}{2 \sqrt{3}}\left(\varepsilon_{11}+\varepsilon_{22}-2 \varepsilon_{33}\right), \\
& e_{4}=\varepsilon_{12}, \\
& e_{5}=\varepsilon_{13}, \\
& e_{6}=\varepsilon_{23},
\end{aligned}
$$

and the elastic compliance matrix and stress become $\overleftrightarrow{s_{T}}$ $=\overleftrightarrow{T} \cdot \overleftrightarrow{s} \cdot \overleftrightarrow{T}^{-1}$ and $\boldsymbol{\tau}=\overleftrightarrow{T} \cdot \boldsymbol{\sigma}$. The polarization already transforms like a $\Gamma_{4}^{-}$distortion so no symmetry adaption is required.

These strain fields are implemented as a boundary condition on cubic samples. Using Voigt notation this is

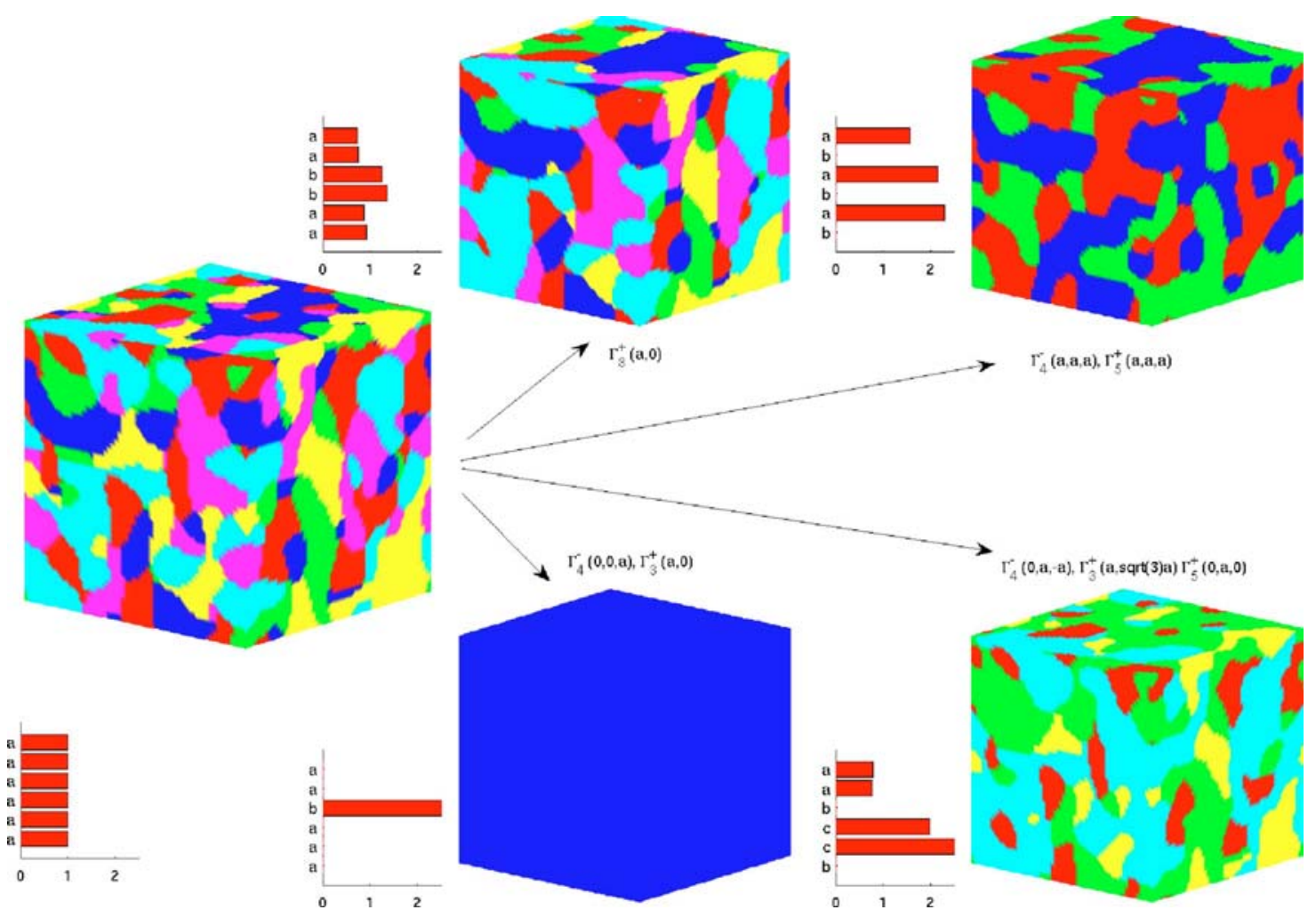

FIG. 1. (Color online) Distinct $(a, 0,0)$-type structures after domain average engineering. 


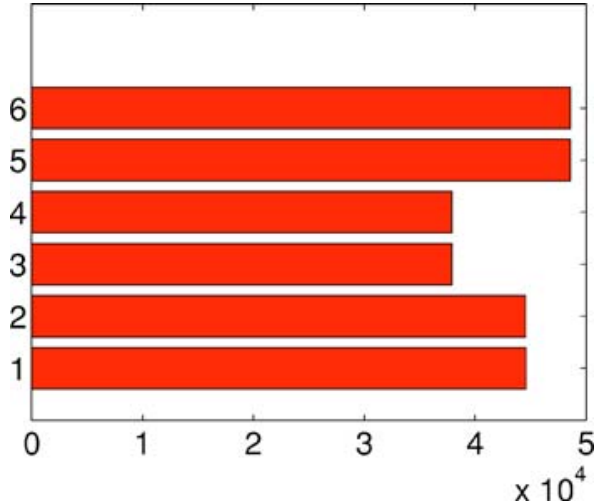

FIG. 2. Original domain fractions in the [100]-ordering structure. Distinct domains are numbered as discussed in Sec. III A 1. The depolarization works only approximately to equalize domain volume fractions.

$$
\left.\mathbf{e}\right|_{\partial \Omega}=\stackrel{\leftrightarrow}{s_{T}} \cdot \boldsymbol{\tau},
$$

where $\Omega$ is the computational volume and $\partial \Omega$ the boundary. A typical run from the paraelectric cubic phase uses periodic boundary conditions in strain and polarization. During DAE, however, polarization is periodic but applied strain is applied to the boundaries as if the simulation supercell were equal to the sample size. This quasiperiodicity is meant to most nearly approach larger simulation sizes and realistic systems.

Although polarization, like strain, is a phenomenon resulting ultimately from atomic displacements, these modes operate at different time scales than strain ${ }^{11}$ and would ideally require different time steps and kinetic coefficients. However, as we seek only to illustrate certain DAE phenomena, we have simplified our treatment for now by setting $\Gamma^{P}=\Gamma^{u}$ and use equal time steps for both types of order parameter. For the system of partial differential equations in Eqs. (7), finite differences and simple explicit Euler integration are used with $\Delta x=0.3$ and $\Delta t=0.001$ or 0.0001 . Taking the functional derivatives leads to the equations shown in Appendix A. As the random force $\xi_{i}^{o p}$ exerts a negligible effect at low temperatures and does not affect the late stages of time evolution we henceforth omit it as has been done elsewhere. ${ }^{11,13}$

\section{RESULTS}

\section{A. Domain average engineering}

With these numerical details established we give a brief introduction to DAE, the subject of this work. Briefly, DAE considers a phase transition where the crystalline structure changes from a high-symmetry group $G$ to a low-symmetry group $H \subset G$. $G$ can be decomposed into cosets,

$$
G=H+g_{2} H+g_{3} H+\cdots+g_{n} H,
$$

where $g_{i}$ are coset representatives and define $n$ equivalent domains associated with this phase transition.

Let us denote each domain volume fraction by $v_{i}$. The action of an operator $g \in G$ on a domain volume fraction results in a transformation from one domain to another,

$$
g v_{i}=v_{j} \quad \text { such that } g g_{i} \in g_{j} H .
$$

Let us write the $n$ domain volume fractions as $\boldsymbol{v}$ $=\left(v_{1}, v_{2}, \ldots, v_{n}\right)$. The action of $g$ on $\boldsymbol{v}$ results in a permutation of the elements of $\boldsymbol{v}$. The set of all distinct transformations of $\boldsymbol{v}$ forms a permutation group $P$. Multidomain symmetry consists of operators $g$ which are mapped onto one of the subgroups of $P$. Since $P$ is a finite group, the number of possible subgroups is also finite and therefore the number of possible symmetries of multidomain structures is finite. Finding the most general form of $\boldsymbol{v}$ for all operators in each subgroup of $P$ which leave $\boldsymbol{v}$ invariant produces the multidomain symmetry groups. For example, suppose $\boldsymbol{v}$ $=(a, a, a, b, b, b)$. This means domains 1,2 , and 3 are present in equal volume fractions and domains 4,5 , and 6 in equal volume fractions but that $a \neq b$. The subgroup of $P$ pertaining to this structure consists of all operators $g$ which leave $\boldsymbol{v}$ invariant.

We use this multidomain volume fraction notation [i.e., $\boldsymbol{v}=(a, a, a, b, b, b)]$ when considering three types of multidomain symmetries: the [100]-ordering $P 4 \mathrm{~mm}$ structure, the [111]-ordering $R 3 m$ structure, and the [110]-ordering Amm2 structure. These are all $\Gamma$-point phase transitions from the $P m \overline{3} m$ phase and so retain the same unit cell. The incipient phase is created by small displacements in the named ordering directions resulting in the spontaneous ferroelectric polarization. The incipient multidomain structure occurs because this displacement is degenerate and could happen in a number of distinct directions. The following three sections discuss each of these cases.

\section{1. [100]-ordering structures}

For the $P m \overline{3} m \rightarrow P 4 m m$ transition, the symmetry is broken by a $\Gamma_{4}^{-}$space group IR and an $(a, 0,0)$ order parameter (OP) direction with six possible energetically equivalent domains. The labelings of the domains follow that of Ref. 21, which are $1(a, 0,0), 2(-a, 0,0), 3(0,0, a), 4(0,0,-a), 5$ $(0, a, 0)$, and $6(0,-a, 0)$. Reference 21 shows that there are 12 possible multidomain structures for such materials. In Table III we have selected 5 of the 12 possible structures. The "Domain set" column shows constants such as $a, b$, and $c$ which again represent volume fractions of each domain.

TABLE IV. 5 of the 17 possible distinct multidomain sets for ferroelectric ordering along [111].

\begin{tabular}{clcccl}
\hline \hline \multicolumn{1}{c}{ Domain set } & \multicolumn{1}{c}{ Group } & $\Gamma_{4}^{-}$ & $\Gamma_{3}^{+}$ & $\Gamma_{5}^{+}$ & \multicolumn{1}{c}{ Connected set } \\
\hline$(a, a, a, a, a, a, a, a)$ & $m \overline{3} m$ & & & $(1,2,3,4,5,6,7,8)$ \\
$(a, b, b, b, a, b, b, b)$ & $\overline{3}_{x y z} m_{\bar{x} y}$ & & & $(a, a, a)$ & $(2,3,4,6,7,8)$ \\
$(a, b, b, a, b, a, a, b)$ & $4_{z} m_{y} m_{\overline{x y}}$ & $(0,0, a)$ & $(a, 0)$ & & $(1,4,6,7)$ \\
$(a, b, b, a, a, b, b, a)$ & $m_{x y} m_{\overline{x y}} m_{z}$ & & $(a, 0)$ & $(a, 0,0)$ & $(1,4,5,8)$ \\
$(a, b, b, b, c, d, d, d)$ & $3_{x y z} m_{\bar{x} y}$ & $(a, a, a)$ & & $(a, a, a)$ & $(2,3,4)$ \\
\hline \hline
\end{tabular}




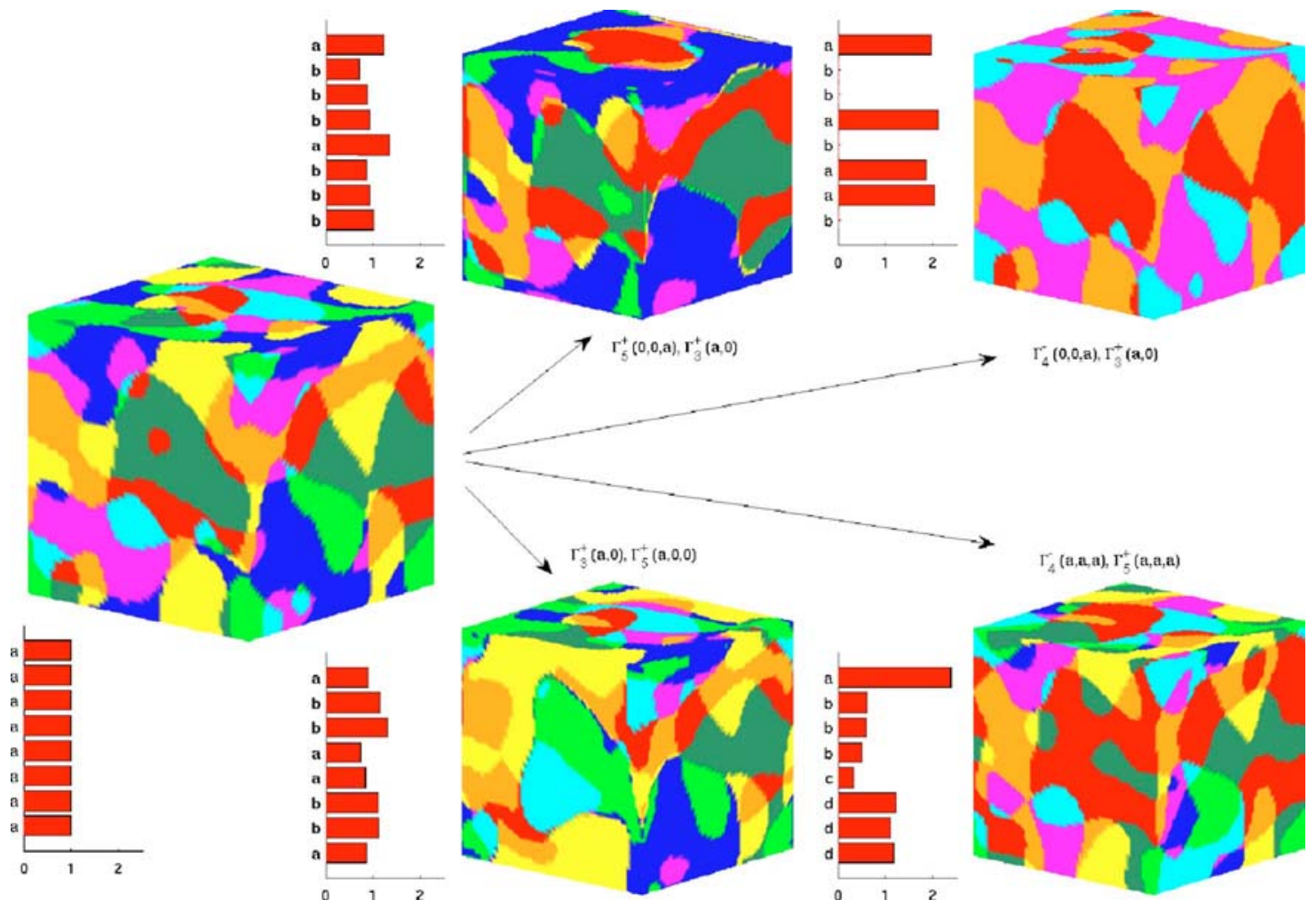

FIG. 3. (Color online) Distinct ( $a, a, a)$-type structures.

The "Group" column gives the point group of the multidomain structure after the convention of Ref. 21. Columns 3-5 give the space group IRs of the external fields and their directions that lead to the domain average engineered structure. The "Connected set" column gives those domains that are equivolume, such as $(1,2,5,6)$ in the previous example and is discussed in detail in Ref. 21.

Figure 1 shows simulations of the multidomain structures given in Table III. The previous equidomain structure is on the far left. Evolved multidomain structures after application of external fields labeled by space group IR and OP direction are on the right. As discussed in Sec. II B, an external electric field has the symmetry of the $\Gamma_{4}^{-}$IR while compressive and shear stresses have the $\Gamma_{3}^{+}$and $\Gamma_{5}^{+}$space group symmetries. In a bar plot near each structure is shown the volume fractions of each of the six domains, numbered from the top. Each domain volume fraction is labeled by that predicted by multidomain group theory. ${ }^{21}$ The agreement is generally good, although many volume fractions that should be equal according to group theory are only approximately equal. This is because the size of the simulation cell, 64 $\times 64 \times 64$, is occasionally comparable to domain size creating artificial boundary effects.

The domain sizes/shapes in this fast quench regime do not always resemble those based on equilibrium experimental conditions or simulations. As discussed, it is impossible, given the size of the simulation box, to achieve the domain volume fraction predicted group theoretically using equilibrium simulations lengths. For this reason this work focuses on the fast quench regime and postpones full equilibrium runs to a later study. When we have let our simulation times approach a greater degree of equilibrium, we have achieved patterns similar to other equilibrium ferroelectric simulations. ${ }^{11}$

In Sec. II B we also discussed how even with a depolarization term the domain volume fractions in the nonengineered multidomain ferroelectric have somewhat unequal domains. This is shown in Fig. 2 for the [100]-ordering structure. To emphasize the changes in the domain volume fractions in the engineered samples we have normalized all volume fractions by this initial ratio. This gives exactly equal domains for the initial cube in Fig. 1 with a magnitude of 1. This practice has been followed in [111]- and [110]-ordering structures as well. When engineering the domains the depolarization term is not included. If it were included, however, we would expect little variation in our results since the depolarization term only approximately equalizes domain volumes as apparent in Fig. 2.

\section{2. [111]-ordering structures}

For the $P m \overline{3} m \rightarrow R 3 m$ transition, the symmetry is broken by a $\Gamma_{4}^{-}$space group IR and an $(a, a, a)$ OP direction with eight possible energetically equivalent domains. The labelings of the domains are $1(a, a, a), 2(a,-a,-a), 3(-a, a$, $-a), 4(-a,-a, a), 5(-a,-a,-a), 6(-a, a, a), 7(a,-a, a)$, and $8(a, a,-a)$. Table IV shows distinct volume fractions for ferroelectric ordering along [111] for 5 of the 17 possible multidomain structures. Figure 3 shows simulations for these types of domain patterns. A $64 \times 64 \times 64$ simulation cell was again used so finite size effects partly come into play, although the agreement between theory and simulation is quite good overall. 
TABLE V. 5 of the 22 possible distinct multidomain sets for ferroelectric ordering along [110]. Only nonzero contributions of the order parameters are shown in columns 3-5.

\begin{tabular}{clllll}
\hline \hline \multicolumn{1}{c}{ Set } & Group & \multicolumn{1}{c}{$\Gamma_{4}^{-}$} & $\Gamma_{3}^{+}$ & $\Gamma_{5}^{+}$ & \multicolumn{1}{c}{ Connected set } \\
\hline$(a, a, a, a, a, a, a, a, a, a, a, a)$ & $m \overline{3} m$ & & & & $(1,2, \ldots 11,12)$ \\
$(a, a, a, a, b, c, c, b, b, c, c, b)$ & $4_{z} m_{x} m_{x y}$ & $(0,0, a)$ & $(a, 0)$ & & $(5,8,9,12)$ \\
$(a, b, b, c, a, b, c, b, a, c, b, b)$ & $3_{x y z} m_{\bar{x} y}$ & & $(a, 0)$ & $(a, 0,0)$ & $(1,4)$ \\
$(a, b, a, b, b, a, a, b, c, c, d, e)$ & $m_{y z} m_{x} 2_{\bar{y} z}$ & $(0, a,-a)$ & $(a, \sqrt{3} a)$ & $(0, a, 0)$ & $(1,3,6,7)$ \\
$(a, b, c, a, d, e, f, g, f, d, g, e)$ & $2_{\bar{y} z}$ & $(a,-a, 0)$ & $(a, 0)$ & $(0, a, a)$ & $(5,10)$ \\
\hline \hline
\end{tabular}

\section{3. [110]-ordering structures}

For the $P m \overline{3} m \rightarrow A m m 2$ transition, the symmetry is broken by a $\Gamma_{4}^{-}$space group IR and an $(a, a, 0)$ OP direction with twelve possible energetically equivalent domains. The labelings of the domains are $1(a, a, 0), 2(a,-a, 0), 3$ $(-a, a, 0), 4(-a,-a, 0), 5(a, 0, a), 6(a, 0,-a), 7(-a, 0$, $-a), 8(-a, 0, a), 9(0, a, a), 10(0,-a,-a), 11(0, a,-a)$, and $12(0,-a, a)$. Table $\mathrm{V}$ shows possible multidomain symmetries for ferroelectric ordering along [110] for 5 of the 22 possible multidomain structures. Figure 4 shows the corresponding simulations for these types of domain patterns. Here a $96 \times 96 \times 96$ simulation cell was used because of the larger number of possible domains. Thus smaller-looking domains are mostly a result of larger system size. Overall, the agreement with theory is quite encouraging, except for structure $2_{\bar{y} z}$ which should have seven distinct domain sizes. Instead domain sizes $d, e$ and $f, g$ appear roughly equal. This appears to be again an artifact of finite system size, especially considering the large number of distinct domains re- quired in the $P m \overline{3} m \rightarrow A m m 2$ case and the good agreement for the previous two cases with smaller numbers of total domains.

\section{B. Discussion}

Figures 1, 3, and 4 show encouraging results regarding the accuracy of group theoretically derived maps of possible multidomain structures in perovskite ferroelectrics. Without any reference to these maps, mesoscopic simulations using combinations of external fields have reproduced predicted symmetries. This represents a significant step in our understanding of mathematical symmetry in complex ceramic structures. Figures 1, 3, and 4 also suggest that the multiorder parameter evolution strategy for ferroelectrics may be more efficient than simulations employing instantaneous mechanical equilibrium. Further testing employing realistic materials will be used to verify this.

The simulation methodology described above has been incorporated in a program (DOMAINS) which is freely available and can describe bulk systems, thin films, and clusters.

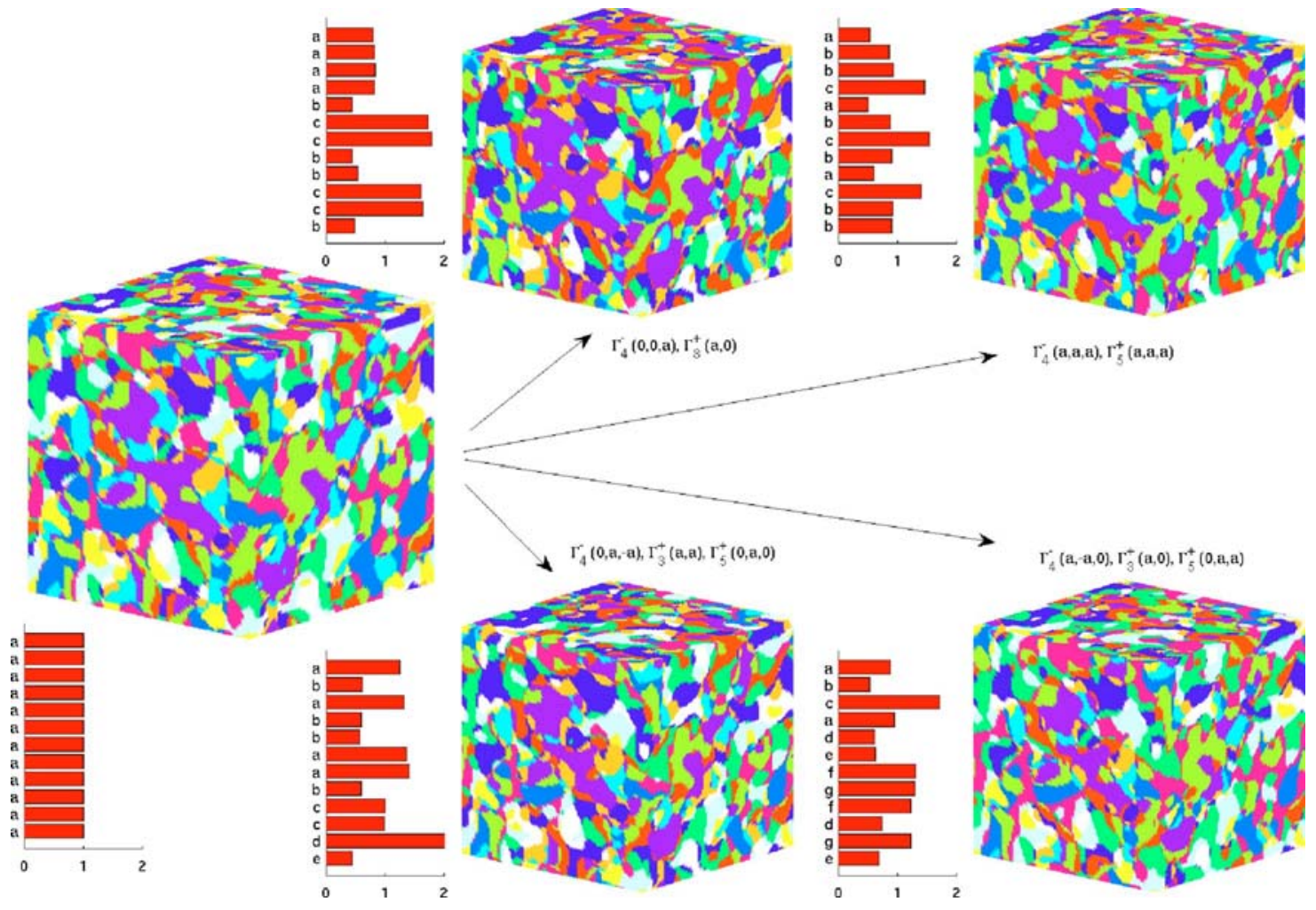

FIG. 4. (Color online) Distinct $(a, a, 0)$-type structures. 
As a possible application of DOMAINS is the processing of MEMS components with small dimensions and nonperiodic boundary conditions; we have avoided spectral methods to numerically solve the differential equations although this is typically the method of choice because of fast Fourier transforms. This real space method allows any type of boundary condition for polarization (charge injected, clamped, periodic) and strain.

Part of the power of phase field theory is that it describes the essential characteristics of microstructure with efficient algebraic expansions. Its limitations are that it is a mean field theory and so lacks interpolarization unit correlations (each mesoscopic unit assigned a polarization is not instantaneously interacting with all other polarization units but only their average). Also, its range of validity is limited to near the phase transition, although this can be improved by adding higher order terms to the expansion. The types of symmetries attainable in the emerging phases are limited to those found in the potential (i.e., antiferroelectric transitions are impossible unless these $X$ point symmetry terms are added to the polarization expansion). To obtain information far from the transition temperature, such as during phase diagram construction, phase field theory must be supplemented with Monte Carlo/molecular dynamics simulations, or lowtemperature expansions.

To further test simultaneous order parameter evolution, DOMAINS is being extended to multiferroics such that ferroelastic transitions compete with and preempt ferroelectric transitions. ${ }^{50}$ Extending to ferromagnetics ${ }^{51}$ would also lead to applications to other multiferroics such as magnetoelectrics. ${ }^{52}$ Other long term extensions might be to ferroelectric nanodomains useful in quantum dot ferroelectric random access memories (FRAMs) or atomic holographic optical storage.

\section{Conclusion}

Encouraging agreement has been observed between a theoretical derivation of possible multidomain structures of three ferroelectric transitions and simulated results. Such transitions occur in many perovskite oxides such as $\mathrm{BaTiO}_{3}$ ([100] ordering), PZN-PT ([111] ordering), and $\mathrm{KNbO}_{3}$ ([110] ordering), and may be systematically applied to such materials in the future. Furthermore, a hybrid multiorder parameter evolution strategy has been used to model threedimensional structures, differing from most previous ferroelectric studies that treat two-dimensional crystals. The group theoretical enumeration of all possible multidomain structures and its numerical validation are needed for high precision domain engineering and are vital to a "bottom-up" micro- and nanotechnology materials design approach to achieve macroscopic materials with precise properties.

\section{ACKNOWLEDGMENTS}

We acknowledge helpful conversations with Allan Jacobs and James Lewis. This work has been supported under DOE Grant No. DE-FG02-03ER46059.

\section{APPENDIX: TIME EVOLUTION EQUATIONS}

After taking the functional derivatives described in Sec. II $\mathrm{B}$, the evolution equations have the following form:

$$
\begin{aligned}
\frac{\partial}{\partial t} P_{1}(\mathbf{r}, t)= & 2 \alpha_{1} P_{1}+4 \alpha_{11} P_{1}^{3}+6 \alpha_{111} P_{1}^{5}+\left(4 \alpha_{11}+2 \alpha_{12}\right) P_{1} P_{2}^{2}+4 \alpha_{112} P_{1} P_{2}^{2}+2 \alpha_{112} P_{1} P_{2}^{4}+4 \alpha_{11} P_{1} P_{3}^{2}+2 \alpha_{12} P_{1} P_{3}^{2} \\
& +4 \alpha_{112} P_{1}^{3} P_{3}^{2}+2 \alpha_{123} P_{1} P_{2}^{2} P_{3}^{2}+2 \alpha_{112} P_{1} P_{3}^{4}-g_{11} \frac{\partial^{2} P_{1}}{\partial x^{2}}-g_{12}\left(\frac{\partial^{2} P_{2}}{\partial x \partial y}+\frac{\partial^{2} P_{3}}{\partial x \partial z}\right)-g_{44}\left(\frac{\partial^{2} P_{1}}{\partial y^{2}}\right. \\
& \left.+\frac{\partial^{2} P_{1}}{\partial z^{2}}\right)+q_{11} \frac{\partial u_{1}}{\partial x}+q_{12} \frac{\partial u_{2}}{\partial y}+q_{44} P_{2}\left(\frac{\partial u_{1}}{\partial y}+\frac{\partial u_{2}}{\partial x}\right)-2 q_{44} P_{3}\left(\frac{\partial u_{1}}{\partial z}+\frac{\partial u_{3}}{\partial x}\right)+E_{1} . \\
\frac{\partial}{\partial t} u_{1}(\mathbf{r}, t)= & c_{11} \frac{\partial^{2} u_{1}}{\partial x^{2}}+c_{44} \frac{\partial^{2} u_{1}}{\partial y^{2}}+c_{44} \frac{\partial^{2} u_{1}}{\partial z^{2}}+\left(c_{12}+c_{44}\right) \frac{\partial^{2} u_{2}}{\partial x \partial y}+\left(c_{12}+c_{44}\right) \frac{\partial^{2} u_{3}}{\partial x \partial z}+2 P_{1}\left(q_{44} \frac{\partial P_{3}}{\partial z}+q_{44} \frac{\partial P_{2}}{\partial y}+q_{11} \frac{\partial P_{1}}{\partial x}\right) \\
& +2 P_{2}\left(q_{12} \frac{\partial P_{2}}{\partial x}+q_{44} \frac{\partial P_{1}}{\partial y}\right)+2 P_{3}\left(q_{44} \frac{\partial P_{1}}{\partial z}+q_{12} \frac{\partial P_{3}}{\partial x}\right) .
\end{aligned}
$$

Evolution equations for other components follow by permutation of the indices.

${ }^{1}$ K. Bhattacharya, A. DeSimone, K. F. Hane, R. D. James, and C. P. Palmstrom, Mater. Sci. Eng., A 685, 273 (1999).

${ }^{2}$ M. H. Dickinson, F.-O. Lehmann, and S. P. Sane, Science 284, 1954 (1999).

${ }^{3}$ M. M. Fejer, G. A. Mangel, D. H. Jundt, and R. L. Byer, IEEE J. Quantum Electron. 28, 2631 (1992)
${ }^{4}$ M. Yamada, Rev. Sci. Instrum. 71, 4010 (2000).

${ }^{5}$ N. G. R. Broderick and G. W. Ross, Phys. Rev. Lett. 84, 4345 (2000).

${ }^{6}$ W. Cao and L. E. Cross, Phys. Rev. B 44, 5 (1991).

${ }^{7}$ R. Ahluwalia and W. Cao, Phys. Rev. B 63, 012103 (2000).

${ }^{8}$ R. Ahluwalia and W. Cao, J. Appl. Phys. 89, 8105 (2001).

${ }^{9}$ R. Ahluwalia and W. Cao, J. Appl. Phys. 93, 537 (2001).

${ }^{10}$ W. Cao, S. Tavener, and S. Xie, J. Appl. Phys. 86, 5739 (1999).

${ }^{11}$ S. Nambu and D. A. Sagala, Phys. Rev. B 50, 5838 (1994).

${ }^{12}$ L. Q. Chen, Annu. Rev. Mater. Res. 32, 113 (2002).

${ }^{13}$ H. L. Hu and L. Q. Chen, Mater. Sci. Eng., A 238, 182 (1997). 
${ }^{14}$ H. L. Hu and L. Q. Chen, J. Am. Ceram. Soc. 81, 492 (1998).

${ }^{15} \mathrm{~W}$. Zhang and K. Bhattacharya, Acta Mater. 53, 185 (2005).

${ }^{16} \mathrm{~W}$. Zhang and K. Bhattacharya, Acta Mater. 53, 199 (2005).

${ }^{17}$ H. T. Stokes and D. M. Hatch, Ferroelectrics 292, 59 (2003).

${ }^{18}$ J. Fousek and L. E. Cross, Ferroelectrics 252, 171 (2001).

${ }^{19}$ J. Fousek, D. B. Litvin, and L. E. Cross, J. Phys.: Condens. Matter 13, L33 (2001)

${ }^{20}$ D. B. Litvin, V. K. Wadhawan, and D. M. Hatch, Ferroelectrics 292, 65 (2003).

${ }^{21}$ D. M. Hatch, H. T. Stokes, and W. Cao, J. Appl. Phys. 94, 5220 (2003).

${ }^{22}$ T. Mitsui, I. Tatsuzaki, and E. Nakamura, An Introduction to the Physics of Ferroelectrics (Gordon and Breach, New York, 1976).

${ }^{23}$ H. T. Stokes and D. M. Hatch, ISOTROPY, 2002; stokes.byu.edu/ isotropy.html

${ }^{24}$ P. C. Hohenberg and B. I. Halperin, Rev. Mod. Phys. 49, 435 (1977).

${ }^{25}$ R. Ahluwalia, T. Lookman, A. Saxena, and W. Cao, Appl. Phys. Lett. 84, 3450 (2004).

${ }^{26}$ J. Wang, S. Q. Shi, L. Q. Chen, Y. Li, and T. Y. Zhang, Acta Mater. 52, 749 (2004).

${ }^{27}$ R. Ahluwalia, T. Lookman, A. Saxena, and S. R. Shenoy, Phase Transitions 77, 457 (2004).

${ }^{28}$ T. Lookman, S. R. Shenoy, K. O. Rasmussen, A. Saxena, and A. R. Bishop, Phys. Rev. B 67, 024114 (2003).

${ }^{29}$ R. Ahluwalia, T. Lookman, and A. Saxena, Phys. Rev. Lett. 91, 055501 (2003).

${ }^{30}$ R. Ahluwalia, T. Lookman, A. Saxena, and R. C. Albers, Acta Mater. 52, 209 (2004).

${ }^{31}$ S. H. Curnoe and A. E. Jacobs, Phys. Rev. B 62, R11925 (2000).

${ }^{32}$ S. H. Curnoe and A. E. Jacobs, Phys. Rev. B 64, 064101 (2001).

${ }^{33}$ S. H. Curnoe and A. E. Jacobs, Phys. Rev. B 63, 094110 (2001).
${ }^{34}$ A. E. Jacobs, S. H. Curnoe, and R. C. Desai, Phys. Rev. B 68, 224104 (2003).

${ }^{35}$ A. E. Jacobs, S. H. Curnoe, and R. C. Desai, Mater. Trans. 45, 1054 (2004).

${ }^{36} \mathrm{P}$. Toledano and V. Dmitriev, Reconstructive Phase Transitions: In Crystals and Quasicrystals (World Scientific, River Edge, NJ, 1996).

${ }^{37} \mathrm{~J}$. C. Toledano and P. Toledano, The Landau Theory of Phase Transitions: Application to Structural, Incommensurate, Magnetic, and Liquid Crystal Systems (World Scientific, Teaneck, NJ, 1987).

${ }^{38}$ D. Vanderbilt, Curr. Opin. Solid State Mater. Sci. 2, 701 (1997).

${ }^{39}$ L. Bellaiche and D. Vanderbilt, Phys. Rev. Lett. 83, 1347 (1999).

${ }^{40}$ H. Fu and R. E. Cohen, Nature (London) 403, 281 (2000).

${ }^{41}$ L. Bellaiche and D. Vanderbilt, Phys. Rev. Lett. 84, 5427 (2000).

${ }^{42}$ J. Iniguez and D. Vanderbilt, Phys. Rev. Lett. 89, 115503 (2002).

${ }^{43}$ I. Kornev, H. Fu, and L. Bellaiche, Phys. Rev. Lett. 93, 196104 (2004).

${ }^{44}$ E. Cockayne, Phys. Rev. B 71, 094302 (2005).

${ }^{45}$ K. Bhattacharya and G. Ravichandran, Acta Mater. 51, 5941 (2003).

${ }^{46}$ J. A. Mettes, J. B. Keith, and R. B. McClurg, Acta Crystallogr., Sect. A: Found. Crystallogr. 60, 621 (2004).

${ }^{47}$ J. B. Keith, J. A. Mettes, and R. B. McClurg, Cryst. Growth Des. 4, 1009 (2004).

${ }^{48}$ J. B. Keith and R. B. McClurg (unpublished).

${ }^{49}$ S. C. Miller and W. F. Love, Tables of Irreducible Representations of Space Groups and Co-Representations of Magnetic Space Groups (Pruett, Boulder, CO, 1967).

${ }^{50}$ J. B. Keith and D. M. Hatch (unpublished).

${ }^{51}$ Y. M. Jin, Y. U. Wang, A. Kazaryan, Y. Wang, D. E. Laughlin, and A. G. Khachaturyan, J. Appl. Phys. 92, 6172 (2002).

${ }^{52}$ N. A. Hill, Annu. Rev. Mater. Res. 32, 1 (2002). 